\title{
Interaction of InGa Liquid Alloy Coolant with Gold Coated Optical Materials
}

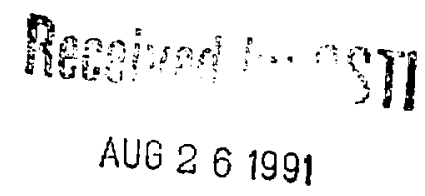

\author{
S. L. Hulbert \\ National Synchrotron Light Source \\ Brookhaven National Laboratory \\ Upton, NY 11973
}

The submitted manuscript has been authored under contract DE-AC02-76CH00016 with the Division of Materials Sciences, U.S. Department of Energy. Accordingly, the U.S. Government retains a non-exclusive, royalty-free license to publish or reproduce the published form of this contribution, or allow others to do so, for U.S. Government purposes.

\section{DISCLAIMER}

This report was prepared as an account of work sponsored by an agency of the United States Government. Neither the United States Government nor any agency thereof, nor any of their employees, makes any warranty, express or implied, or assumes any legal liability or responsibility for the accuracy, completeness, or usefulness of any information, apparatus, product, of process disclosed, or represents that its use would not infringe privately owned rights. Reference herein to any specific commercial product, process, or service by trade name, trademark, manufacturer, or otherwise does not necessarily constitute or imply its endorsement, recommendation, or favoring by the United States Government or any agency thereof. The views and opinions of authors expressed herein do not necessarily state or reflect those of the United States Government or any agency thereof. 


\title{
Interaction of InGa Liquid Alloy Coolant with Gold Coated Optical Materials
}

\author{
S. L. Hulbert \\ National Synchrotron Light Source \\ Brookhaven National Laboratory \\ Upton, NY 11973
}

\begin{abstract}
A significant reaction is reported between gold surfaces and an indium-gallium eutectic liquid alloy often used to transfer heat away from optical elements in high power synchrotron radiation beamlines. This reaction proceeds at the slightly elevated temperatures $\left(70^{\circ} \mathrm{C}\right)$ typical of conservative bakeouts used to achieve ultrahigh vacuum in the chambers which house these optical elements (mirrors, gratings, and crystals) without damaging their highly precise figure and finish.
\end{abstract}

The nature and extent of this reaction is discussed, based mostly on the experience gained recently during the vacuum commissioning of two mirror chambers for a VUV wiggler-based synchrotron radiation beamline. 


\section{Introduction}

For the last decade or so, liquid indium-gallium eutectic alloy has been used as an efficient heat transfer medium from optical elements (e.g. mirrors, crystals, and gratings) to water-cooling blocks in the ultra-high vacuum environments of synchrotron radiation beamlines. The eutectic is gallium-rich ( $\mathrm{Ga} 75.5 \%$, In $24.5 \%$ ), melts at $15.7^{\circ} \mathrm{C}$, and has a negligibly small vapor pressure $\left(-10^{-12} \text { Torr at } 300^{\circ} \mathrm{C}\right)^{1}$. Successful implementation of cooling schemes using this alloy requires that InGa not interact significantly with the surfaces it contacts. For example, aluminum is a very poor choice of mirror or cooling block material because the gallium in these alloys is known to react rather strongly with aluminum via grain boundary diffusion at room temperature and above ${ }^{2}$. In 1983 , Hunter and Williams ${ }^{1}$ demonstrated the dramatic extent of the Ga-Al reaction, which extends to a depth of $2.5 \mathrm{~mm}$ below the aluminum surface, causing severe embrittlement: the surface turns to a grayish powder and flakes

away. Hunter and Williams reported the results of a multi-year study ${ }^{1}$ of the interactions of these indium-gallium eutectic alloys with a variety of different metal surfaces, at room and elevated $\left(300^{\circ} \mathrm{C}\right)$ temperatures. In addition to the strong $\mathrm{Ga}-\mathrm{Al}$ reaction, the conclusions of their study were as follows: (1) The slight reaction of these alloys with $\mathrm{Cu}-\mathrm{Be}$, brass, $\mathrm{Si}, \mathrm{Ag}$, and $\mathrm{Mg}$ is most likely slow enough that their use with these metals is acceptable. That is, they don't significantly diffuse aways from the contact area, either laterally or into the bulk material. (2) The Ga-Cu reaction is stronger than with the metals listed above, so contact between InGa eutectic alloy and copper is not recommended. (3) No measurable interaction was fuund between InGa eutectic alloys and W, 304 S.S., Ni, graphite, pyrolytic carbon, Ge, Invar, SiC, B, Pb, Pt, or Rin.

\section{Mirror results and discussion}

The purpose of this note is to report the existence of a rather strong reaction between InGa eutectic alloy and Au at approximately $70^{\circ} \mathrm{C}$ both in ultrahigh vacuum 
and at atmospheric pressure. This reaction was discovered during the initial lowtemperature $\left(70^{\circ} \mathrm{C}\right)$ bakeout of the fully-assembled $M_{0}$ and $M_{1}$ mirror boxes for the wiggler beamline $113 \mathrm{U}-\mathrm{A}$ on the VUV storage ring at the NSLS.

The $\mathrm{M}_{0}$ mirror box contains three Au-coated $(900 \AA)$ SiC mirrors $(350 \times 50 \times 35$ $\mathrm{mm}^{3}$ ) and $\mathrm{M}_{1}$ contains one Au-coated $(900 \AA) \mathrm{SiC}$ mirror $\left(500 \times 60 \times 35 \mathrm{~mm}^{3}\right)$. All four mirror segments are cooled by water-cooled Cu plates $(-1 " \times 1 / 4 " \times$ length of mirror) which are spring loaded against the long sides of each mirror segment as close as practical $(\leq 1 \mathrm{~mm})$ to the mirror front surface, with a very small amount ( $\leq 1$ drop) of InGa alloy spread out uniformly in between as the thermal transfer medium. The side of the $\mathrm{Cu}$ block facing the mirror is Ni-coated to avoid $\mathrm{Ga}-\mathrm{Cu}$ reaction. Also, the Au-coating on the $\mathrm{SiC}$ mirror extends down the unpolished sides of the mirror where the $\mathrm{Cu}$ cooling plates make contact. It turns out to be significant that $\mathrm{M}_{0}$ is a horizontally deflecting mirror (the $M_{0}$ surface lies in a vertical plane) and $M_{1}$ is a vertically deflecting mirror (the $M_{1}$ surface lies in an horizontal plane).

As shown in Fig. 1, the surfaces of the horizontally-deflecting $\mathbf{M}_{0}$ mirror segments suffered only minor diffusion of Ga across the mirror surface, extending at most $-5 \mathrm{~mm}$ from its starting location at the edges of the mirror surface. This diffusion occurred during the initial $70^{\circ} \mathrm{C}$ bakeout of these mirrors and chamber, which lasted for approximately two days. Fig. 1 shows the maximal extent of the Ga-Au reaction for the amount of $\mathrm{Ga}$ in the system, because subsequent bakeouts (at $70^{\circ} \mathrm{C}$ ) have not expanded the reacted surface area. On the other hand, $-25 \%$ of the surface area of the vertically-deflecting $M_{1}$ mirror, shown in Fig. 2 , is covered by Ga-Au reaction product, which extends completely across the width of the mirror in a couple of places. Since no greater thickness of the InGa layer was used for $M_{1}$ compared to $M_{0}$ (and the bakeout temperature was the same), it must be concluded that orienting a mirror surface perpendicular to gravity (mirror surface in an horizontal plane) enhances this 
reaction.

Mirror $\mathrm{M}_{1}$ has been subsequently stripped and recoated with $\mathrm{Pt}$, and the thermal contact is made with a thin indium foil rather than InGa alloy. This conservative treatment may not be necessary, since the Ga-Pt reaction has been shown to be negligible ${ }^{l}$. During the stripping process, the entire Ga-Au reaction product ( $\mathrm{Ga}-\mathrm{Au}$ alloy) lifted from the $\mathrm{SiC}$ substrate as a film, indicating that the full thickness of the Au coating $(900 \AA)$ was reacted with $\mathrm{Ga}$ in the affected regions of the mirror surface.

\section{Test slide results and discussion}

In order to understand the nature of the Ga-Au reaction, three Au-coated (400 $\AA$ thickness) glass microscope slides $\left(1 / 2^{\prime \prime} \times 1 / 2^{\prime \prime}\right)$ were subjected to the following treatments:

Treatment 1: a drop of InGa alloy was placed near the middle of a slide and baked to $75^{\circ} \mathrm{C}$ in vacuum $\left(-10^{-3}\right.$ Torr $)$.

Treatment 2: same as treatment 1 , except the vacuum vessel was backfilled to approximately 600 Tor: of dry nitrogen before baking.

Treatment 3: a drop of InGa alloy was placed near the middle of a slide and allowed to sit in air at room temperature.

For this study, the orientation of the slides relative to gravity was not varied: all three slides were kept in an horizontal position.

The treatment 1 slide began to expand after one hour at elevated temperature $\left(75^{\circ} \mathrm{C}\right)$ and tontinued to expand for the next two days, at which point the heat was turned off. The original drop of InGa alloy had expanded to a diameter of $12 \mathrm{~mm}$, nearly covering the microscope slide. The treatment 2 slide began to expand sometime within the first three hours at elevated temperature $\left(75^{\circ} \mathrm{C}\right)$ and continued to expand for the next three days, at which point the heat was turned off. Again, the drop of InGa 
alloy had expanded to a diameter of $12 \mathrm{~mm}$. The treatment 3 slide did not show visible changes for three weeks in air at room temperature. Clearly, elevated temperature $\left(70-75^{\circ} \mathrm{C}\right)$ is sufficient to initiate and maintain a significant interaction between InGa alloy and $\mathrm{Au}$, and there is no visible dependence on pressure (at atmospheric pressure or below).

In order to determine the nature of the interaction of the InGa alloy with the Au layer, scanning electron microscopy (SEM) with x-ray elemental analysis was performed on the three slides after treatment. A representative SEM photograph of the outside edge of the reacted area of the treatment 1 slide is shown in Fig. 3. The InGa "puddle" was expanding from the upper right to the lower left in Fig. 3 when the heat was turned off. The front edge of the expanding InGa puddle is marked by the lower left edge of the dark region (the narrow path running from upper left to lower right), and contracted to the upper right edge of the dark region upon cooling from $75^{\circ} \mathrm{C}$ to room temperature, leaving a small "mountain" of InGa in one spot. The lower left region of Fig. 3 is unreacted Au surface, as determined by $x$-ray elemental analysis, which shows only the characteristic emission lines of $\mathrm{Au}$, the glass substrate $(\mathrm{Si}, \mathrm{Ca})$, and $\mathrm{Cr}$ (which is used as a binder to prevent gold island formation). X-ray elemental analysis of the dark region reveals the presence of substrate, $\mathrm{Cr}$ binder, and unreacted InGa alloy, but no evidence of $\mathrm{Au}$. This indicates that InGa alloy reacts completely with a $400 \AA \mathrm{Au}$ layer at $75^{\circ} \mathrm{C}$ and that the reaction products (presumably In and $\mathrm{Ga}$ Au alloy) cling to the InGa puddle as it contracts during cooling. The main InGa puddle (upper right portion of Fig. 3) and the island left in the dark region show only In and $\mathrm{Ga} \mathrm{x}$-ray emission lines in the ratio characteristic of bulk InGa alloy. Similar SEM topography and elemental composition was observed around the exterior of the InGa puddle created via treatment 2.

A somewhat different and more instructive situation arises around a small 
droplet of InGa alloy which was inadvertently deposited in a region of the treatment 1 slide which was destined to lie outside the maximum extent of the expanding InGa puddle. The CEM photograph of this droplet after being subjected to treatment 1 is shown in Fig. 4. The edge of the large InGa "puddle" can be seen at the top of this figure. The region outside the oval-shaped rings is unreacted Au layer, while the dark region surrounding the odd-shaped bright features in the center of the oval shows mainly glass substrate (Si,Ca) x-ray emission lines, binder $(\mathrm{Cr})$ lines, and a small fraction of unreacted alloy (In,Ga) lines, identical to the dark region in Fig. 3. The concentric oval-shaped rings which surround the odd-shaped bright features and the dark region can be observed, apparently, because the amount of InGa in this droplet was small enough that it did not expand to cover its own reaction front. $\mathrm{X}$-ray elemental analysis of these rings show that they consist of $\mathrm{Ga}$ and $\mathrm{Au}$, probably as an alloy reaction product; only a small fraction of substrate lines are observed, and there is no measurable In intensity above background. The indium, which does not react strongly with gold, remains concentrated in some of the odd-shaped bright features in the center of the oval region; these features exhibit mostly In and Au x-ray emission lines. Others of the bright features show mainly $\mathrm{Ga}$ and $\mathrm{Au}$ lines, much like the oval-shaped concentric rings, and are assigned to active $\mathrm{Ga}-\mathrm{Au}$ reaction zones.

For comparison, a small drop of InGa alloy was placed on a clean, unpolished $\mathrm{Cu}$ surface and baked $\left(150^{\circ} \mathrm{C}\right)$ in vacuum $\left(10^{-3}\right.$ Torr $)$ for three days. Recall that in the study by Hunter and Williams ${ }^{1}$, copper interacted more strongly with InGa than any other material studied except aluminum. In contrast to the gold results described above, the drop of InGa on a copper substrate did not expand significantly. Fig. 5 is an SEM photograph of the boundary of the drop, which shows the unexpanded drop in the upper left corner, a granular reaction region covering most of the figure, and bare copper surface in the lower right corner. X-ray elemental analysis of the granular 
reaction zone shows $\mathrm{Ga}$ and $\mathrm{Cu}$ emission lines but no indium lines, which indicates that, as for gold and aluminum, the InGa- $\mathrm{Cu}$ reaction is dominated by the $\mathrm{Ga}-\mathrm{Cu}$ reaction.

\section{Conclusions}

InGa eutectic alloy is an ideal thermal transfer medium in uitrahigh vacuum environments, except for the reactive properties of its $\mathrm{Ga}$ component. The list of unacceptable mating materials has now been increased by one ( $\mathrm{Au}$ ), and the list of acceptable materials has been reiterated (see Introduction). Of course, any materials not on these lists that might come in contact with InGa eutectic alloy should be checked for unacceptably strong reaction before use.

\section{Acknowledgements}

The expert technical assistance provided by A. Lenhard during assembly of the mirror holders, installation of the mirrors, and the microscope slide tests is gratefully acknowledged. The SEM photographs and elemental $\mathrm{x}$-ray analysis were provided by R. Sabatini. This work was supported by the Division of Material Sciences U. S. Department of Energy under Contract No. DE-AC02-76CH00016.

\section{References}

1. W. R. Hunter and R. T. Williams, Nucl. Instrum. Methods 222, 345 (1984), and references therein.

2. W. R Groggin and J. W. Moberly, Trans. Am. Soc. Metals 59, 315 (1966).

\section{Figure Captions}

1. Normal incidence photograph of the central portio of one of the three segments of the horizontally-deflecting Au-coated $(900 \AA)$ CVD SiC $\mathrm{M}_{0}$ mirror $(350 \mathrm{~mm} \times$ 
$50 \mathrm{~mm}$ surface) as installed at NSLS beamline U13U-A. Note the reflection of the camera and the eight-inch viewport through which the photograph was taken. The water-cooled copper blocks are spring-loaded against the top and bottom faces of the mirror by stainless steel pins held in a large aluminum U-channel. Note that small semi-circular shaped regions of the surface have reacted with the InGa alloy which forms the interface between the mirror and the top copper cooling block.

2. End (a) and side (b) perspective photographs of the vertically-deflecting Au-coated (900A) CVD SiC $M_{1}$ mirror ( $500 \mathrm{~mm} \times 60 \mathrm{~mm}$ surface), after being removed from its chamber and holder for stripping and recoating. Note that InGa alloy has reacted with a large fraction $(\sim 25 \%)$ of the Au-coated mirror surface.

3. SEM photograph of the edge of a "puddle" of InGa alloy on a Au-coated (400§) microscope slide created by baking $\left(75^{\circ} \mathrm{C}\right)$ a small drop of $\mathrm{InGa}$ in vacuum $\left(10^{-3}\right.$ Torr) for two days. Baking in dry nitrogen (600 Torr) produces similar results. The puddle was expanding from the upper left to the lower right in this photograph when the heat was tumed off. The front edge of the expanding InGa puddle contracted from the lower left edge to the upper right edge of the dark region (the narrow path running from upper left to lower right) upon cooling from $75^{\circ} \mathrm{C}$ to room temperature, leaving a small "mountain" of InGa in one spot. The lower left region of the photograph is unreacted Au surface. X-ray elemental analysis of the dark region shows no evidence of $\mathrm{Au}$, indicating that InGa reacts completely with a $400 \AA$ Au layer at $75^{\circ} \mathrm{C}$. $200 \times$ magnification, $100 \mu \mathrm{m}$ scale bar shown.

4. SEM photograph of a small droplet of InGa alloy which has reacted with a Aucoated $(400 \AA)$ microscope slide by baking $\left(75^{\circ} \mathrm{C}\right)$ in vacuum $\left(10^{-3}\right.$ Torr) for two days. The edge of the large InGa "puddle" shown in Fig. 3 is shown at the top of this photograph. The amount of InGa in this droplet was small enough that it did not expand to cover its own reaction front, which appears as a set of concentric oval-shaped rings surrounding the bright odd-shaped remains of the initial droplet. 
$X$-ray elemental analysis of the concentric oval rings shows no evidence of indium, indicating that the Ga-Au reaction dominates the interaction of InGa alloy with Au. The unreactive indium is left concentrated in some of the odd-shaped bright features in the center of the oval region, which exhibit a thirty-fold increase in the ratio of In to $\mathrm{Ga}$ x-ray emission intensity compared to bulk unreacted InGa alloy. $500 \times$ magnification, $10 \mu \mathrm{m}$ scale bar shown.

5. SEM photograph of a small drop of InGa alloy which has reacted with a clean, unpolished $\mathrm{Cu}$ surface by baking $\left(150^{\circ} \mathrm{C}\right)$ in vacuum $\left(10^{-3} \mathrm{Torr}\right)$ for three days. In contrast to the Au surface (Figs. 3 and 4), the drop does not expand significantly; the boundary of the InGa "puddle" in the upper left corner of the photograph is close to its original location. The InGa-Cu reaction is dominated by the $\mathrm{Ga}-\mathrm{Cu}$ reaction: $x$-ray elemental analysis of the granular reaction zone (which covers most of the area of photograph) shows $\mathrm{Ga}$ and $\mathrm{Cu}$ emission lines but no indium lines. $300 \times$ magnification, $100 \mu \mathrm{m}$ scale bar shown. 


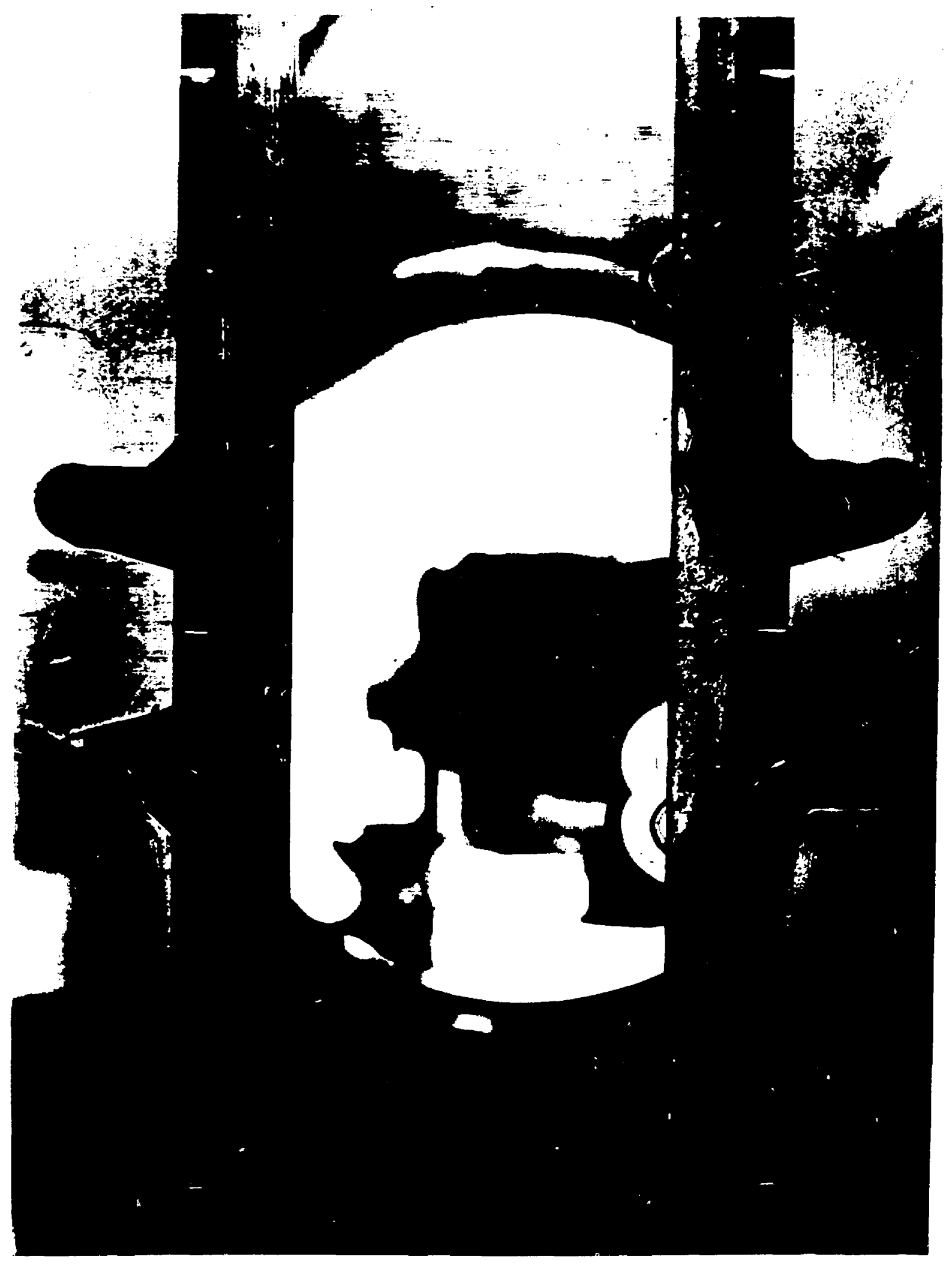




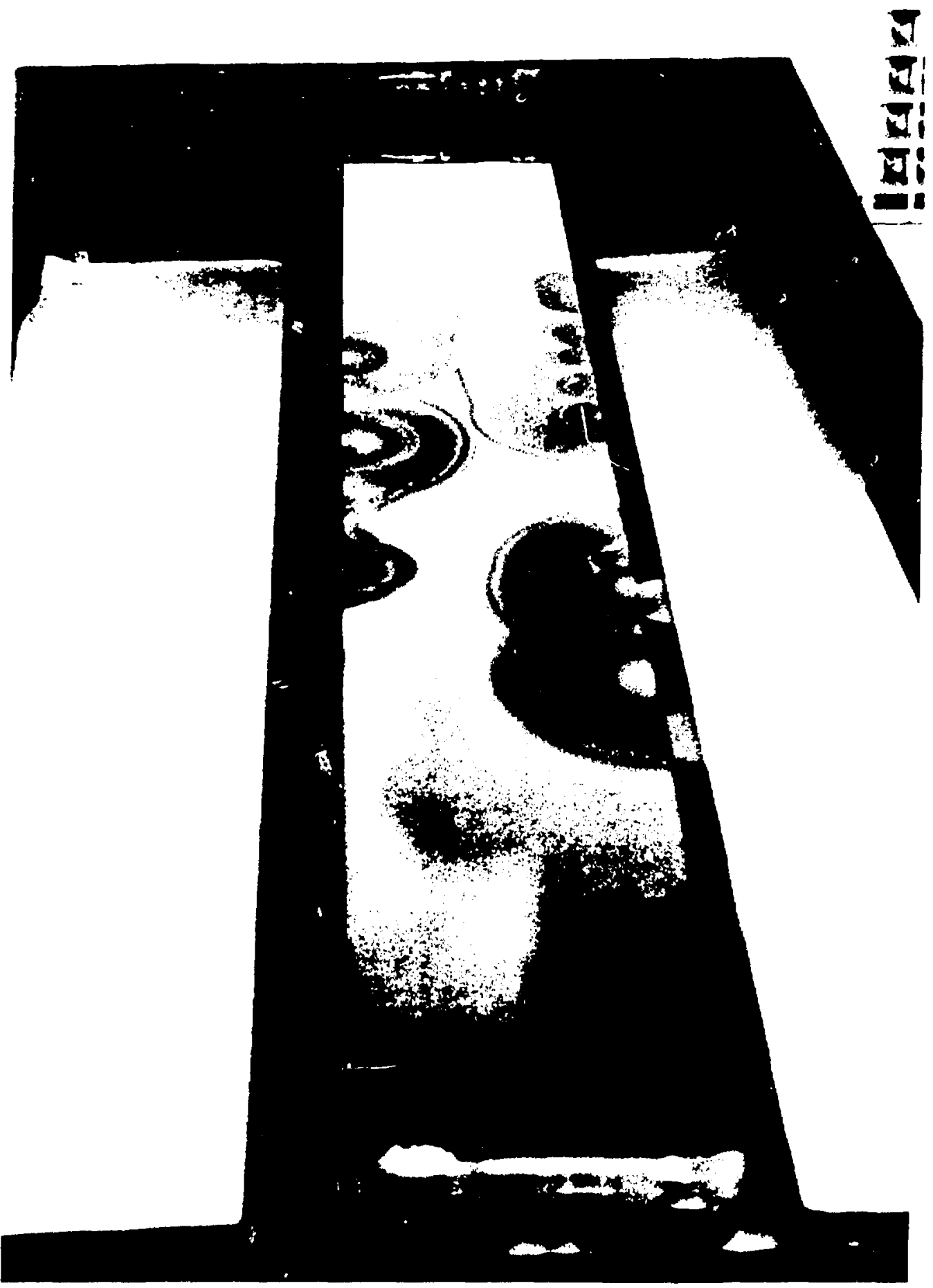




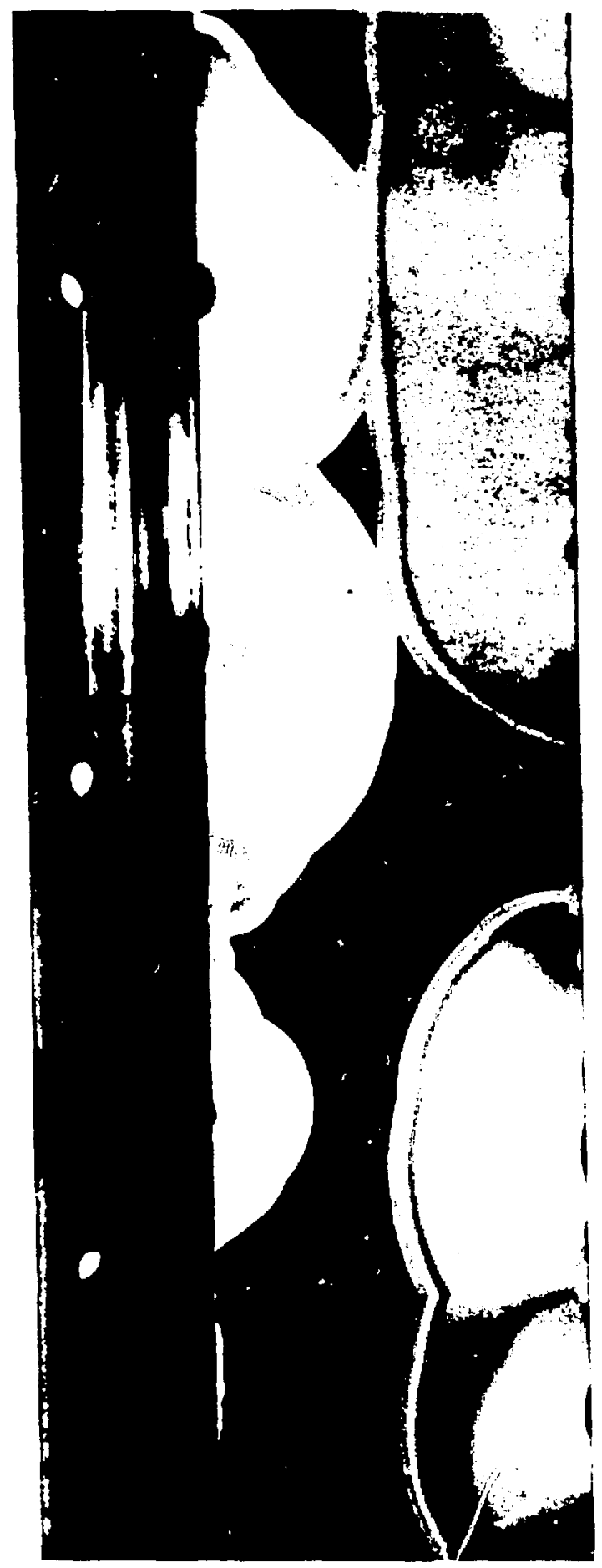




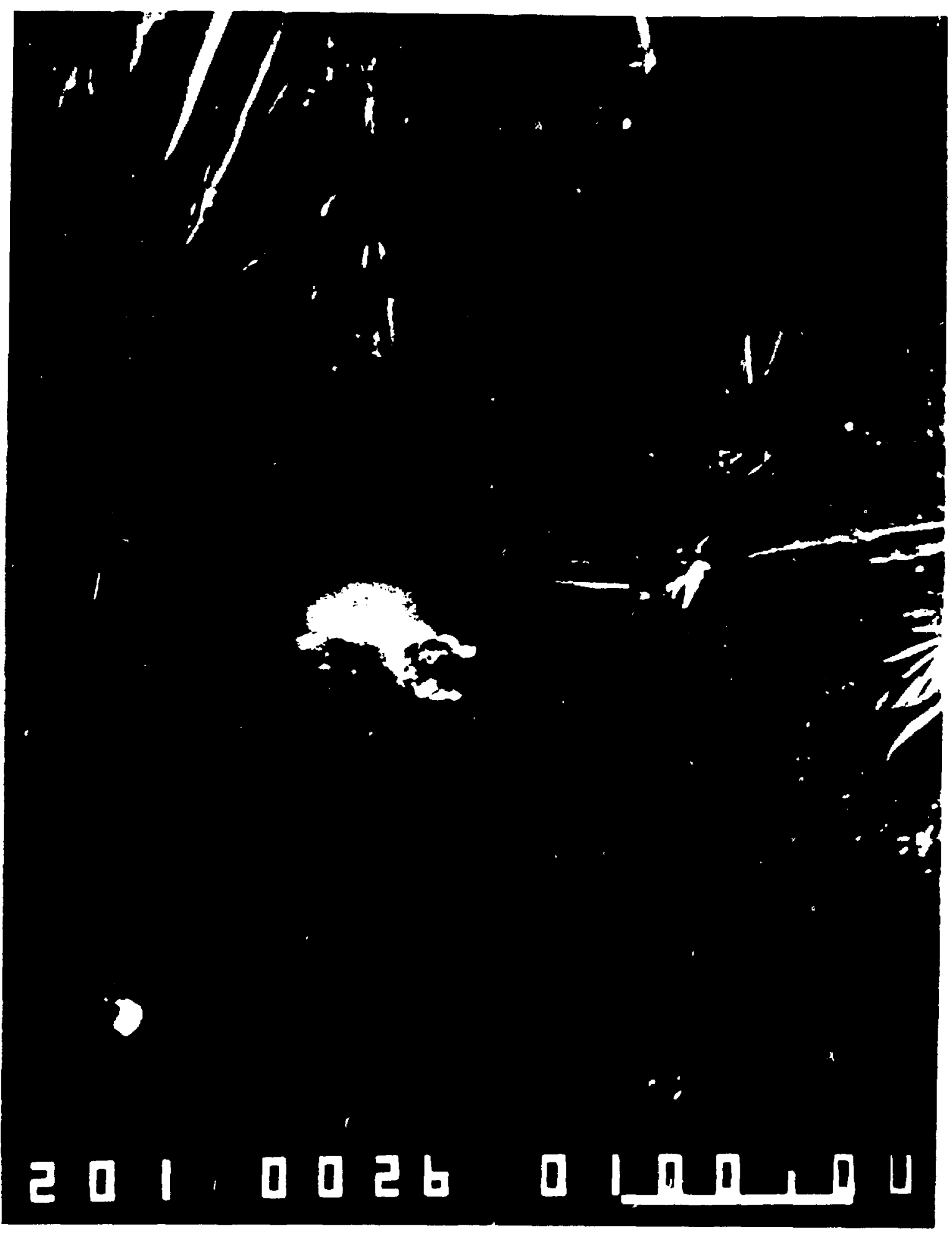




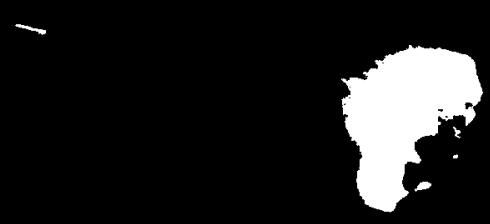

4

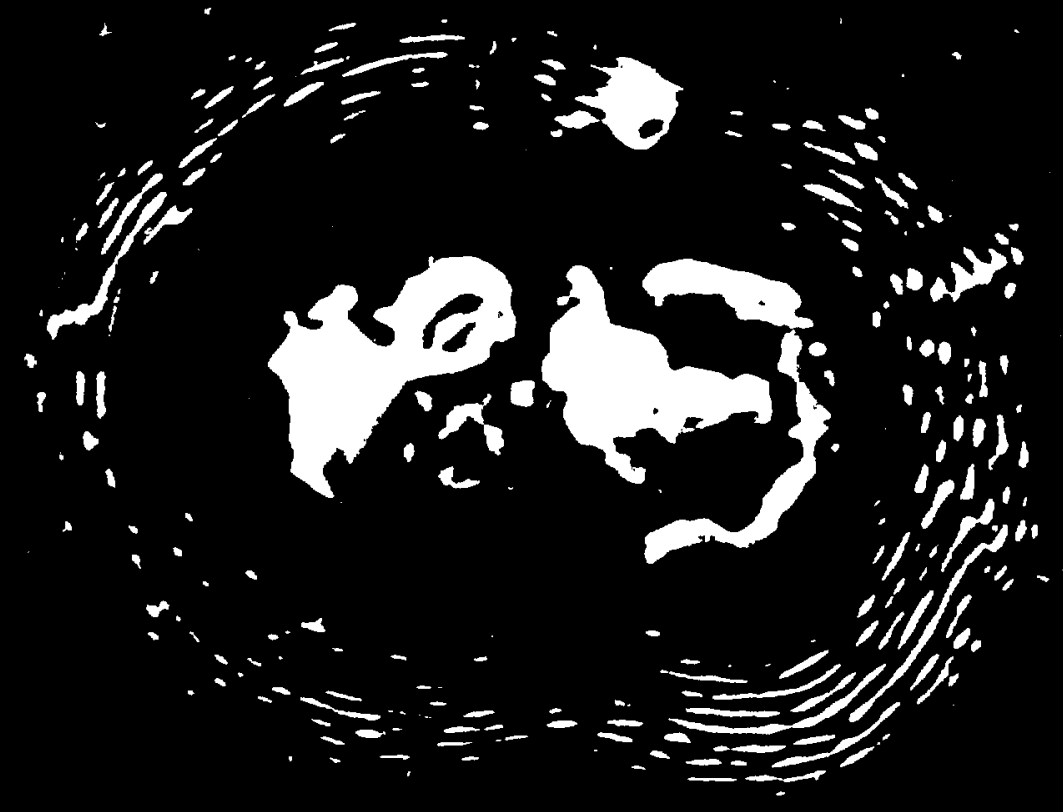




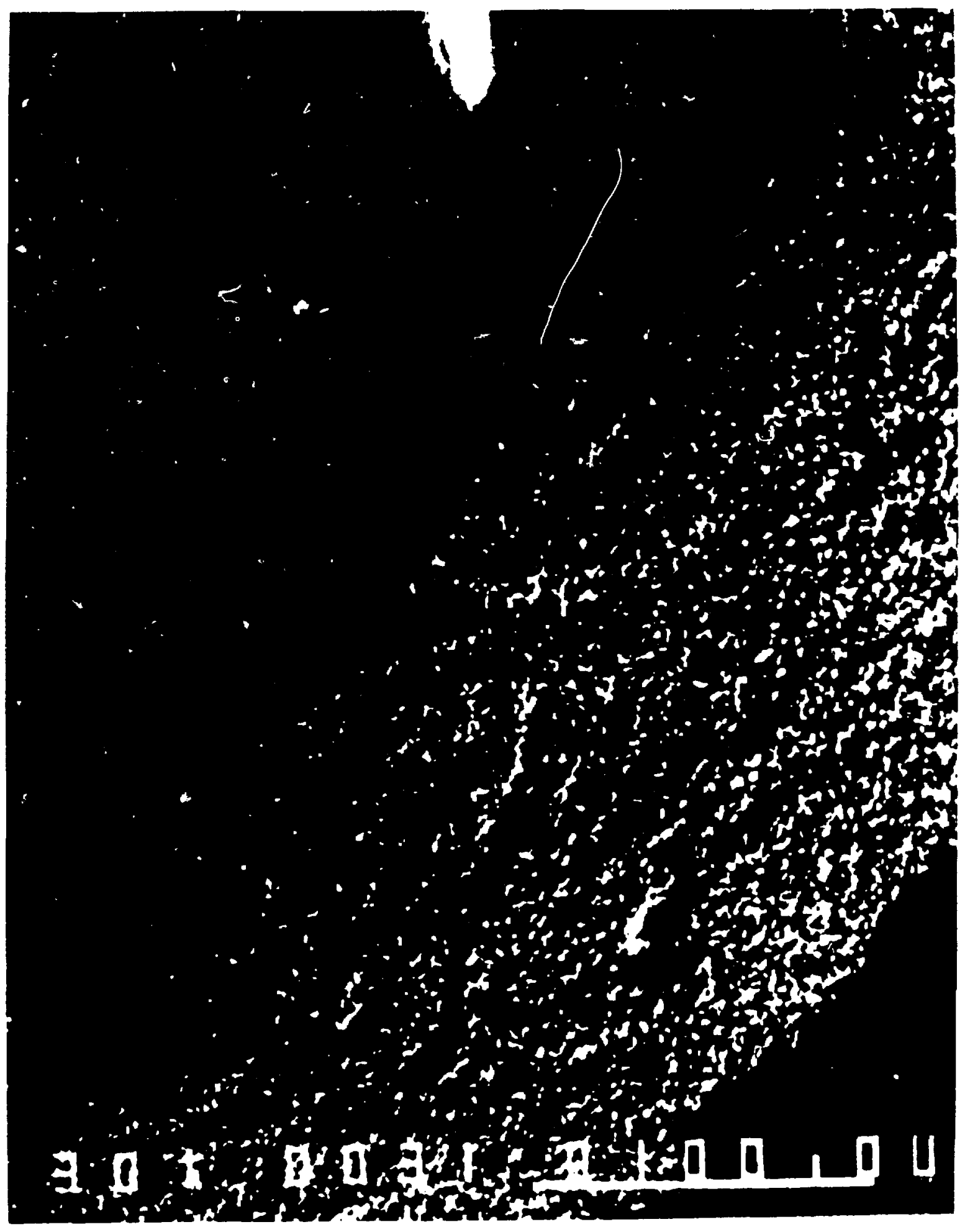

